\title{
Integrated Spectra of Early-Type Galaxies
}

\author{
James A. Rose ${ }^{1}$ \\ ${ }^{1}$ University of North Carolina at Chapel Hill
}

\begin{abstract}
I review efforts to determine the ages and chemical compositions of early-type galaxies from their integrated spectra. After reviewing the history of integrated light analyses, we consider how reliably the light-weighted mean ages and chemical compositions of galaxies can be extracted from current state-of-the-art population synthesis modelling and observational data. Particular attention is given to assessing the best strategies for pursuing integrated spectrum analysis, both from observational and modelling standpoints. Finally, we consider efforts to move beyond light-weighted mean ages and metallicities, specifically, to constrain star formation histories with multiple episodes of star formation.
\end{abstract}

\section{Introduction}

The intention of this talk is to review the subject of integrated spectra of early-type galaxies. Given the wide scope of such a review, I will take the following more limited approach. I begin with a historical look at the subject of integrated spectra over the past few decades, since a great deal of useful perspective can be attained from seeing how we arrived at our present state of knowledge. Then I will pose a few basic questions:

o How reliable are the current methods for extracting light-weighted mean age and abundance information from integrated spectra of early-type galaxies?

o What are the optimum strategies for determining ages and chemical compositions? For example, what is the optimum set of spectral indices or full-spectrum fitting methods? What is the optimum combination of spectral resolution, wavelength coverage, and $\mathrm{S} / \mathrm{N}$ ratio required? And what are the relative merits of studying a few galaxies in great detail versus attaining lower $\mathrm{S} / \mathrm{N}$ ratio for large samples of galaxies?

o What can we expect in the near future from advances in both population synthesis modelling and observational capabilities to move the field forward? Specifically, can we move beyond determinations of light-weighted mean ages and abundances to infer the presence of multiple populations, i.e., map out star formation histories? And how far can we pursue 2-dimensional spatial mapping of the populations in early-type galaxies out to low surface brightness regions?

To answer all of these questions would require a truly lengthy review, thus the goal of my talk is to least bring up the questions, and then make some attempt to answer them, or at least express some opinions.

\section{Historical Review}

My starting point for integrated spectra of early-type galaxies is the work of Beatrice Tinsley in the late 60's and early 70's, in which she showed through basic population synthesis arguments that the luminosity evolution of elliptical galaxies as a function 
of redshift affects the magnitude-redshift diagram of bright Eg's more drastically than that caused by the geometry of the expanding universe (Tinsley 1972). Consequently, to determine our cosmological world model via the magnitude-redshift requires accurate modelling of the luminosity evolution of galaxies. While Tinsley's work was not the only reason at the time to pursue the subject of integrated light of galaxies, it certainly provided a major impetus to the field. Over the subsequent three decades, progress in the interpretation of integrated spectra of Eg's has relied on several developments:

1) Advances in detector technology enabled the acquisition of reliable digital spectrophotometry of galaxies by, e.g., Spinrad \& Taylor (1971), O'Connell (1976), and Gunn, Stryker, \& Tinsley (1981), initially with multi-channel scanners developed at Lick Observatory (Wampler 1966) and at Palomar Observatory Oke (1969). This was followed by the commissioning of the higher resolution Image Dissector Scanner (IDS) at Lick (Robinson \& Wampler 1972), which led to the development of the Lick spectral index system (e.g., Burstein et al. 1984).

2) In addition to acquiring spectra, a system of measuring key spectral properties needed to be developed. Early work by Spinrad et al. (1971) focussed primarily on low resolution measurement of the most prominent spectral features, e.g., $\mathrm{CN}, \mathrm{Mg}, \mathrm{Na}$, and Balmer lines. As higher resolution spectra became available, Faber and collaborators revolutionized the field by developing the Lick spectral index system (Burstein et al. 1984; Faber et al. 1985), which to this day represents a bedrock of integrated spectrum studies.

3) On the theoretical side, a crucial advance was the development of "evolutionary synthesis" modelling, i.e., the application of full astrophysical constraints to the modelling of integrated spectra. As has been described by other reviewers at this conference, this "simple stellar population" approach relies on combining a set of isochrones with an empirical or theoretical library of stellar spectra to synthesize the spectrum of a stellar population. With the completion of the Yale isochrones (Mengel et al. 1979), SSP's could be modelled and compared to spectra of Eg's. As is further described later, spectral libraries were unfortunately slow to develop, in particular with regard to metal-poor stars. Until the mid-80's, spectral libraries in fact tended to follow 2-dimensional MKK classification only.

4) Finally, for a field to advance it requires the stimulus of an important, and controversial, result. In this regard I single out the landmark O'Connell (1980) paper, in which he proposed that the visible spectrum of the small Eg M32 is dominated by intermediateage $(\sim 5$ Gyr $)$ stars. This result naturally carries profound implications for our views of $\mathrm{Eg}$ formation and evolution. Another result that generated great interest is the Burstein et al. (1984) paper, in which they found that Galactic globular clusters (GC's), M31 GC's, and Eg's each formed separate sequences in key color and spectral index plots.

By the mid-1980's, the study of galaxy integrated spectra had pretty well evolved into its currently recognizable form in terms of observational and modelling techniques. In debating the issue of intermediate-age stars in M32, the necessity of disentangling age from metallicity effects was being recognized and worked on. The pair of articles by O'Connell (1986) and Renzini (1986) in the STScI Symposium on "Stellar Populations" provides an interesting summary of the discussions that were ongoing at that time.

However, towards the end of the decade, a huge bump in the road appeared in the form of the non-solar abundance ratios (NSAR) observed in massive Eg's. In retrospect, this problem had been present for a long time, having been demonstrated by Peterson (1976) and O'Connell (1976), and strongly hinted at in the early work of Spinrad \& Taylor (1971). It was certainly as well a part of the above-mentioned Renzini (1986) contribution at the STScI meeting. However, the problem generated a major new impact 
beginning with the $\mathrm{PhD}$ thesis of Peletier (1989), continuing on with, e.g., Worthey et al (1992), Vazdekis et al (1997), Trager et al (2000), Kuntschner et al (2001), Thomas, Maraston, \& Bender (2003). The effort involved in sorting out the NSAR problem certainly complicated the approach to determining galaxy ages and abundances from their integrated spectra. On the other hand, it led to a much more careful analysis of the problem, specifically, concerning what element abundances key spectral indices are actually measuring, that has been of overall benefit to securing reliable answers in the long run. Overcoming the limitations of spectral libraries based on Solar Neighborhood stars, with their limited set of abundance ratios eventually required the modelling of abundance ratio changes through synthetic spectra based on model atmospheres. This crucial work for the Lick spectral index system was carried out by Tripicco \& Bell (1995), thus representing an essential step forward. As well, efforts have been ongoing to model the effects of NSAR on stellar interiors, first through the use of scaled solar isochrones, and more recently through stellar models based on NSAR (e.g., Thomas, Maraston, \& Bender 2003; Korn, Maraston, \& Thomas 2005; Lee \& Worthey 2005).

At the same time that NSAR effects became widely recognized, the age-metallicity degeneracy issue took on a new degree of focus with the massive paper by Worthey (1994), in which he showed how nicely the degeneracy can be broken by judicious choice of a Balmer line index (specifically, $\mathrm{H} \beta$ ) plotted versus a metal-line index. Thus by the end of the 1990's, the NSAR situation and age-metallicity degeneracy issue appeared to both be quite well in hand when a second major bump in the road appeared: the ages for old stellar populations as derived from their integrated spectra were unreasonably large, much older than the age of the universe. Again, this situation was apparent for some time, but was drawn into sharp focus by Gibson et al. (1999), who showed that the age for the "metal-rich" Galactic globular cluster 47 Tuc obtained from analysis of its integrated spectrum far exceeded that obtained from fitting its well-determined color-magnitude diagram (CMD) with stellar isochrones. A resolution of this problem has been mostly achieved. Vazdekis et al. (2001), in considering NSAR effects, the effects of element diffusion, and a refined definition of the $\mathrm{H} \gamma$ line strength, greatly alleviated the extent of the spectroscopic versus CMD age discrepancy. Schiavon et al. (2002a,b) provided further resolution to the problem by noting that the observed giant branch luminosity function in the core of 47 Tuc exceeds the numbers predicted by theoretical isochrones at a level above the horizontal branch. Once the GB LF is properly accounted for in the models, the spectroscopic age of 47 Tuc is significantly decreased. This work highlights just how difficult it is to achieve an accurate zeropoint age from models of integrated spectra. CMD-based ages basically just have to get the overall shape of the isochrone correct at the main sequence turnoff (MSTO). In contrast, since all phases of the HR diagram contribute to the integrated spectrum, the shape of the isochrone has to be correct at all phases, as well as the luminosity function! The above studies certainly underscore the necessity of fully testing the population synthesis models on "known" stellar systems, such as globular clusters. Fortunately, high S/N ratio integrated spectra of a large sample of Galactic globular clusters are now publicly available (Schiavon et al. 2005).

A major development of the past few years has been the production and public release of three large new spectral libraries which cover a much greater range of atmospheric parameters than previously (Prugniel \& Soubiran 2001; Valdes et al. 2004; SanchezBlazquez et al. 2006a). To return to this issue, the main problem for producing such spectral libraries had been the lack of fundamental data on the atmospheric parameters of a sufficient number of stars in the Solar Neighborhood. A solution to this has come (1) from the heroic work of Cayrel de Strobel \& Soubiran over the years to compile 
catalogs of stars with determined parameters and (2) from high resolution spectroscopic abundance analyses of large samples of stars by, e.g., Edvardsson et al. (1993), which provided a rich new set of targets for the spectral libraries. The Edvardsson et al (1993) study of late $\mathrm{F}$ and early $\mathrm{G}$ dwarfs was particularly momentous, given that it provides a large number of target stars that represent the MSTO of old stellar populations for a variety of abundances.

Additional developments that brings us to the present day are the ongoing work to improve the publicly available stellar isochrones, and the development of easy-to-use population synthesis models, which incorporate the isochrones with the stellar libraries. As well, new approaches to full-spectrum fitting (as opposed to extracting age information from a few specific indices) provide promising avenues for optimized analyses of integrated spectra (e.g., Madgwick et al. 2002; Panter et al. 2003; Ocvirk et al. 2006). Finally, grids of synthetic spectra, based on model atmospheres with a variety of abundance ratios, and covering a large spectral region, are rapidly progressing (Coelho et al. 2005). All of these advances are reviewed elsewhere in this conference.

\section{Examination of Spectra}

Before turning to the set of questions laid out in the Introduction, it is worthwhile first to look at a few stellar and galaxy spectra, to obtain some initial perspective on the problem of measuring properties of galaxies in integrated light. Here we do this at higher resolution than is generally carried out in galaxy work, due to the limitation imposed by the high velocity dispersion in massive galaxies; seeing particularly the blue spectrum at higher resolution can be an important guide to designing a strategy of measuring the information present in the lower resolution spectra.

In Fig. 1 two spectra are plotted from the Indo-US library of coude feed stellar spectra, at a resolution of $\sim 1.2 \AA \mathrm{FWHM}$. This figure illustrates the $\mathrm{T}_{\text {eff }}$-metallicity degeneracy of stellar spectra in the blue, which translates into an age-metallicity degeneracy in integrated spectra of stellar populations. Despite having very similar spectra, the top star has $[\mathrm{Fe} / \mathrm{H}]$ approximately 0.6 dex higher than the bottom star. It also demonstrates how crowded the blue spectral region is, and consequently how difficult it is to make a clean measurement of any single spectral feature.

In Fig. 2 another issue is illustrated in a plot of three stars with G0 spectral type but different luminosity classes. Note that the differences between the dwarf and giant star, in particular, are quite subtle, with the most substantial change with luminosity class (i.e., surface gravity) demonstrated by the $\operatorname{SrII} \lambda 4077$ feature that is marked in the figure. The point of this figure is that key information can be carried by even a single spectral feature, which, particularly in the blue, can be easily lost among the multitude of other features which tend to be redundant in the message that they carry.

Finally, the integrated spectrum of the low $\sigma$ galaxy M32 is compared to that of the more massive NGC 821 in the blue in Fig. 3. The first thing to notice is the apparent weakening of the lines in NGC 821 relative to M32, due to the greater velocity broadening. Clearly, it is essential to know the velocity dispersion, as well as the spectral resolution, to correctly model the detailed line structure in the observed spectrum. A second issue is the presence of the CN molecular feature, which extends from the bandhead at $4216 \AA$ to $\mathrm{H} \delta$ at $4101 \AA$. This feature, which is extremely difficult to model in massive Eg's, due to NSAR effects, can compromise indices that purport to measure both $\mathrm{H} \delta$ and the Ca I feature at $4226 \AA$, through "contamination" of the pseudo-continuum passbands and/or the feature itself (Prochaska et al. 2005, 2006). The point here is that one has to be extremely careful, especially in the blue, regarding what specific features are present in 


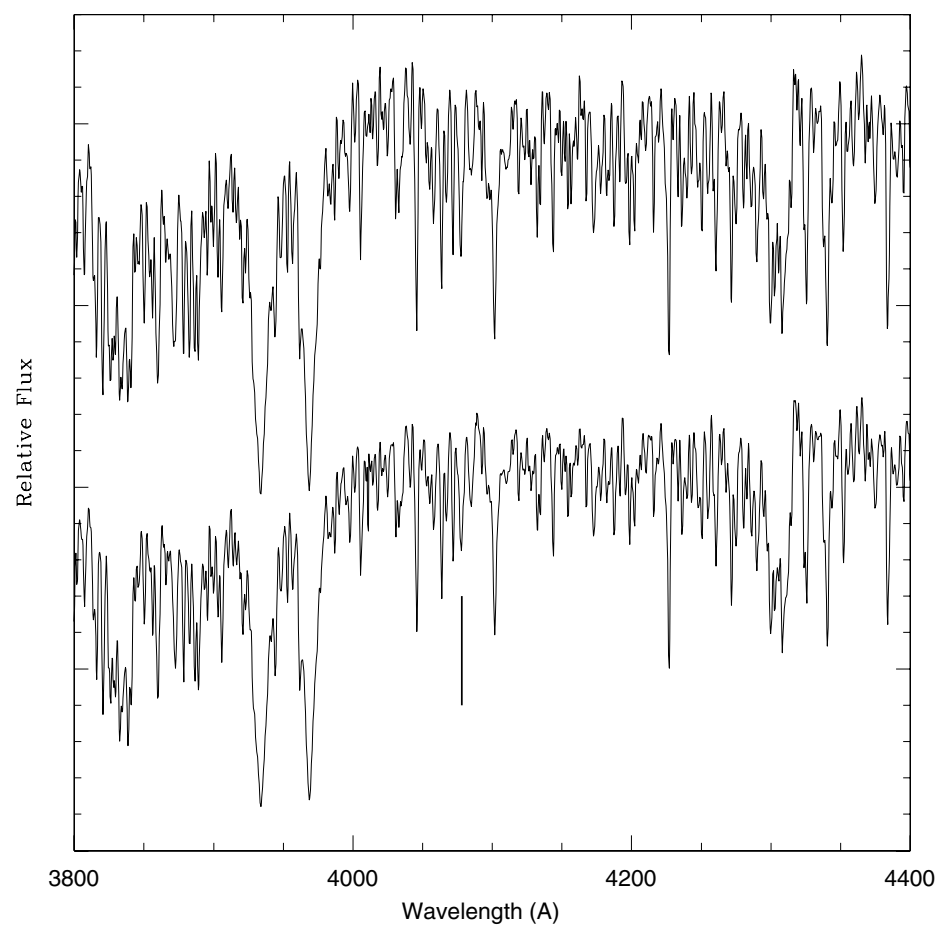

Figure 1. The spectrum of the metal-rich star HD30562 (top spectrum), with $[\mathrm{Fe} / \mathrm{H}]=+0.14$, is compared with that of the metal-poor star HD157214 (bottom spectrum), with $[\mathrm{Fe} / \mathrm{H}]=-0.41$. The remarkable similarity of the two spectra illustrates the degenerate effects of $\mathrm{T}_{\text {eff }}$ and $[\mathrm{Fe} / \mathrm{H}]$ on the appearance of the spectra.

the region of the spectral feature of interest, given the fact that NSAR issues complicate the accurate modelling of integrated spectra.

\section{Reliability of Integrated Spectrum Analysis}

Several extensive studies of the light-weighted mean ages and metallicities of earlytype galaxies have been completed within the past few years, which on the whole lead to a consistent picture regarding the star formation and chemical evolution histories of nearby galaxies. First, Trager et al. 2000, Caldwell, Rose \& Concannon 2003, and Sanchez-Blazquez et al. 2006b find that for field early-type galaxies there is a spread in light-weighted mean age at all galaxy masses (as traced by the velocity dispersion, $\sigma$ ), but that the spread in ages among low $\sigma$ galaxies is substantially greater than that for high $\sigma$ galaxies. Most lower mass Eg's have had more extended SF histories and slower CE than their higher mass counterparts. On the other hand, Kuntschner (2000) finds no evidence for an age spread in ellipticals in the Fornax cluster; instead, a welldefined mass-metallicity relation is seen. Several studies (Kuntschner et al. 2002; Thomas et al. 2005; Nelan et al. 2005; Sanchez-Blazquez et al. 2006b) find that E/S0 galaxies are younger and more metal-rich in low density environments than in clusters.

The above results appear to be quite robust, based in each case on two or more independent studies in terms of the galaxy data. Furthermore, it is encouraging that similar results are obtained from higher order Balmer lines as from $\mathrm{H} \beta$ (Caldwell, Rose \& Concan- 


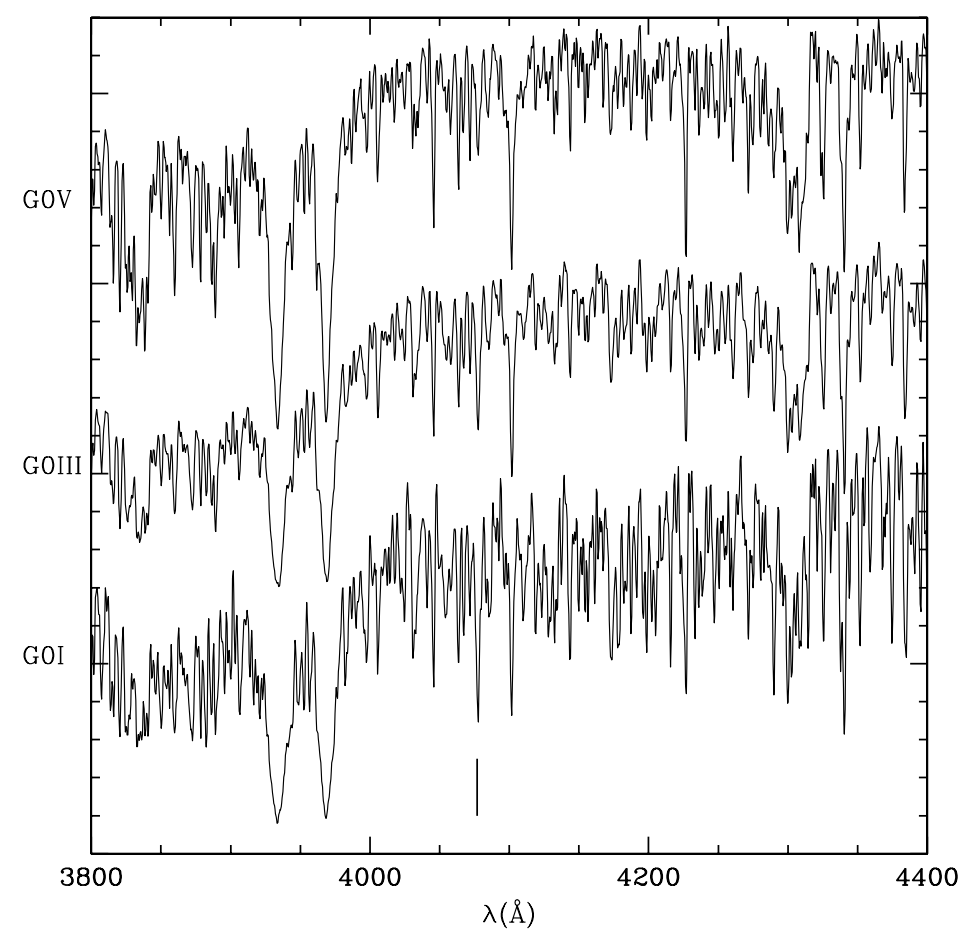

Figure 2. The spectra of three G0 stars with differing luminosity classes are compared, again in the restricted blue wavelength region. From top to bottom they are the G0V star HD184499, the G0III star HD111812, and the G0Ib star HD204 867. The singly ionized SrII $\lambda 4077$ line, which shows strong sensitivity to luminosity class (i.e., surface gravity), is specifically marked in HD204867.

non 2003). Naturally, they suffer from the uncertainties in age and chemical composition zeropoints in the stellar populations models that have been so extensively summarized elsewhere in this conference. Thus, it is the comparative statements, i.e., between low and high density environments, and between low and high $\sigma$ galaxies, that are what need to be taken seriously. Overall, these results provide some important constraints on large-scale numerical modelling of galaxy evolution.

There have been a couple of lingering uncertainties regarding the case for intermediate age stars in the integrated light of galaxies. The first concerns the possibility that the enhanced Balmer lines first discovered by O'Connell (1980) in M32, and seen in so many other early-type galaxies, could be due to a "contaminating" population of blue horizontal branch stars (e.g., Lee, Yoon \& Lee 2000), or to blue straggler stars, or to a very small young star component. However, as originally presented in Rose (1985), and further discussed in Caldwell, Rose \& Concannon (2003), Leonardi \& Rose (2003), and Trager et al. (2005), strong constraints can be placed on the presence of hot stars by using an index that measures the depths of the Ca II lines relative to $\mathrm{H} \epsilon$. These studies find in general that a hot star component cannot account for the Balmer line strengths observed in M32 and other ellipticals. There has also been some concern that the blue end of the red horizontal branch (i.e., F giants) could produce the enhanced Balmer line strengths. However, Rose $(1985 ; 1994)$ has ruled out that possibility based on a spectral index that measures the mean surface gravity of the integrated spectrum. 


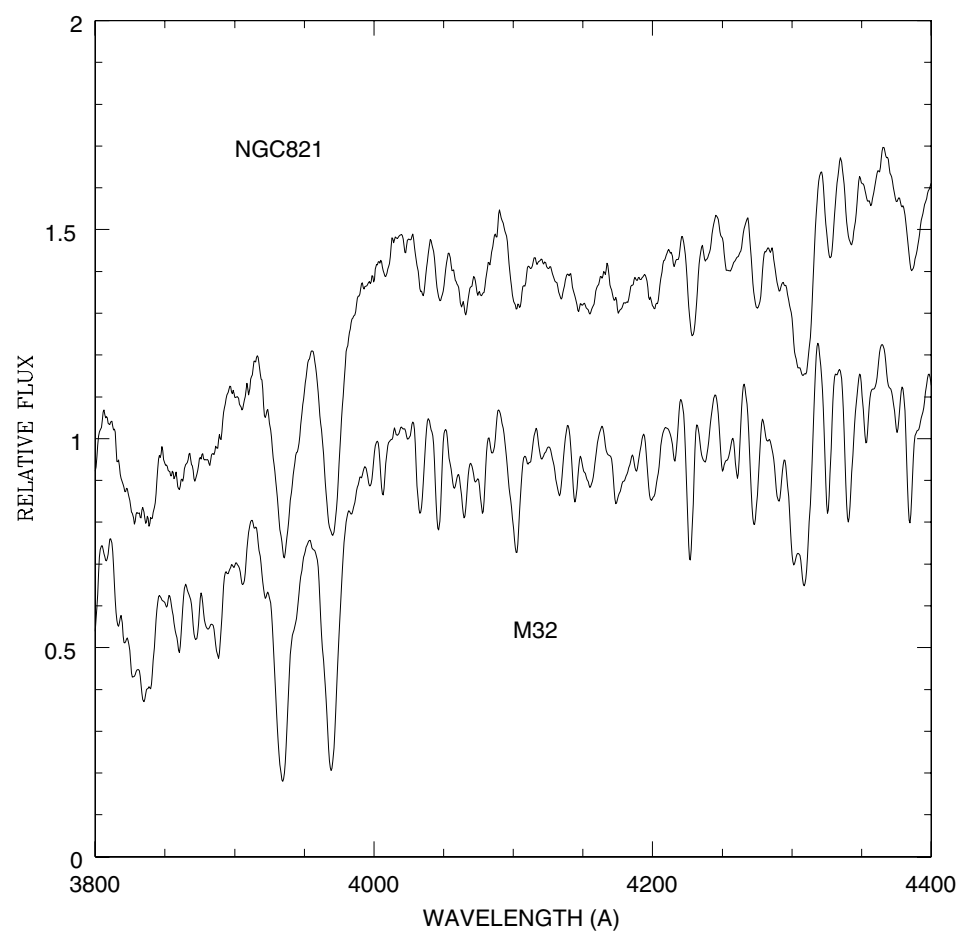

Figure 3. The integrated spectrum of the low $\sigma$ galaxy M32 is compared to that of the higher $\sigma$ galaxy NGC 821

Regarding zeropoints, note the latest results reported by TANTALO!!

\section{Optimum Strategies for Integrated Spectrum Analysis}

\subsection{Spectral Resolution versus Spectral Coverage}

In designing an optimum approach to extracting information from integrated spectra, a starting point is to consider the balance between wavelength coverage and spectral resolution. Clearly, increasing wavelength coverage is always desirable, given that the relative contributions from different regions of the HR diagram are highly wavelength dependent, but comes at the expense of loss in spectral resolution. As was pointed out earlier, an accurate measure of both spectral resolution and velocity broadening is necessary in the blue if small-scale fitting of individual features is to be achieved. Unfortunately, spectrographs suffer from temperature-dependent focus variations with wavelength. A suggestion, then, is to take the spectra at higher wavelength resolution than necessary; in smoothing to the working resolution, small focus variations in the original spectrum will become unimportant. In addition, while Nyquist sampling (2 pixels per resolution element) in principle is sufficient if the noise is well-behaved, it is worthwhile to overresolve spectral features, given the multiple interpolations that take place in spectral data reductions.

In short, is it better to maximize the number of spectral features covered (which may carry much redundant information) or to optimize the precision of fewer features by oversampling a smaller wavelength region? Striking this balance is dependent on the age 
precision desired, and also depends greatly on whether one is considering young (a few broad lines) versus old (many crowded/overlapping features) integrated spectra. Finally, I must admit to a bias towards the optical spectrum, dominated by the best understood stars (MS, SGB, RGB) as opposed to the UV and IR, dominated by small numbers of the hottest and coolest stars, all in uncertain evolutionary stages. On the other hand, use of the AGB transition at $\sim 100 \mathrm{Myr}$ as an age indicator for younger populations is clearly a promising approach, especially for working at high redshift with JWST (e.g., Mouhcine \& Lancon 2003; Maraston 2005).

\subsection{Spectral Indices versus Full Spectrum Fitting}

Another key question is whether age and chemical composition are best extracted from analysis of a few individual spectral indices (e.g., Lick index system), or via one of the full-spectrum fitting approaches. In principle, all of the spectrum is useful, thus it makes little sense to throw away any spectral information. On the other hand, as demonstrated in Fig. 2, there is huge redundancy in the spectrum, thus it is important not to swamp out the special signal carried by one or a few features with an overabundance of other lines. As recognized in Panter et al. (2003), in effect the issue is that of optimum weighting of spectral information when following a full spectrum approach. One can achieve this via spectral compression in the MOPED approach (Panter et al. 2003), principal component analysis (PCA, e.g., Madgwick et al. 2002; Ferreras et al. 2006), or ANN (see Singh et al. 2006 for application to stellar spectral classification). Rather than attempt a major discussion of the spectral index versus full spectrum approach, I'll simply pose several basic questions for the fitting approach. First, how sensitive are the results (i.e., extracted ages and metallicities) to slight errors in spectral resolution and/or wavelength zeropoint? Second, how do errors in the SSP models translate into errors in the results? Specifically, what, if any, problems arise in using a solar abundance ratio model to fit an observed NSAR galaxy spectrum? An additional concern is mainly directed at the PCA approach. Since spectral evolution is so non-linear with age (the difference between a 1 Gyr spectrum and a 4 Gyr spectrum is huge compared to that between a 4 Gyr and a 10 Gyr spectrum), any approach based on finding orthogonal basis spectra seems pre-disposed to see intermediate age populations as a combination of very young and very old populations. I expect that this pre-disposition would apply to the MOPED approach as well, given that it fits the observed spectrum with a series of SSPs that are logarithmically spaced in time. On the other hand, the PCA approach is well suited to cases in which a recent poststarburst population is superposed on an older population (see the Wild et al. poster from this conference). Finally, a word of encouragement to full spectrum fitters to compare their results carefully to those found from specifc indices, and, where they differ, try to understand why they do so.

\subsection{Detailed Individual Studies versus Large Samples}

A final question regarding methods is whether more can be learned from detailed studies of a few galaxies, as opposed to lower quality data on very large samples. There are indeed benefits to very extensive studies of a single galaxy. If high enough $\mathrm{S} / \mathrm{N}$ ratio spectra are obtained over a sufficient wavelength region, then two things become possible. First, the abundance pattern becomes measurable, yielding $\mathrm{Fe}, \mathrm{Ca}, \mathrm{N}, \mathrm{C}$, and $\mathrm{Mg}$, and perhaps more. Second, through measuring age and metallicity sensitive features at different wavelengths, it in principle becomes possible to discern two-, or even multi-, population models as distinct from a single light-weighted mean age (see §6.1). In addition, spatially resolved spectroscopy provides key information about the location, and hence origin, of younger populations. On the other hand, there are clearly limits to how 
much information can be extracted about the star formation and chemical enrichment history of a single galaxy, no matter how high the $\mathrm{S} / \mathrm{N}$ ratio is of the integrated spectrum. A powerful approach is to obtain data on large samples, in which case the evolutionary histories of galaxies can be constrained by comparing the observed distributions of galaxies in various color and spectral index diagrams to parametrized models of galaxy star formation histories. Trager et al. (2000) used an basic approach of this kind to argue for a two-population ("frosting") model for the younger light-weighted ages in their sample. The power of combining local samples with those at higher redshifts is illustrated in the recent Faber et al. (2005) analysis of the blue and red sequences of galaxies at both low and intermediate redshift. The data towards evolution of galaxies from the blue onto the red sequence through both wet and dry mergers.

\section{Near-Future Prospects}

In closing, it is interesting to consider what advances might take place over the next few years. A few ideas are briefly discussed below.

\subsection{Multi-Population Analysis}

As discussed in $\S 4$, there tends to be good agreement among recent studies concerning the light-weighted mean ages and metallicities of galaxies, what might be referred to as first order population synthesis. The challenge that now awaits is to somehow extract real star formation histories, beginning with the simplest problem of discriminating the case of a galaxy with two episodes of star formation from that of a uniform age galaxy. The latter represents the first step in second order population synthesis. As is discussed in, e.g., Schiavon et al. (2004) and Sanchez-Blazquez et al. (2006b), the key to detecting a young and old population mix is in the fact that the young population contributes proportionately more light at bluer wavelengths than it does at redder wavlengths. Hence, age indicators at different wavelengths should produce systematically different light-weighted ages. While straightforward in principle, in practise it is full of pitfalls. Specifically, it becomes essential to sort out the zeropoint problems of each individual spectral index utilized; as mentioned above, NSAR can lead to real problems in modelling specific indices correctly. Producing optimum, and well-understood, indices for the Balmer lines is thus of central importance to the success of second order population synthesis. For recent work on $\mathrm{H} \beta$, see the Cervantes \& Vazdekis poster from this conference; for earlier work on optimizing $\mathrm{H} \gamma$, see Vazdekis et al. (2001). In short, while the multi-population approach can be expected to make progress over the coming few years, and in fact is naturally built into most full-spectrum fitting methods, it will take awhile to establish secure results.

\subsection{Spatial Mapping of Stellar Populations}

Another promising step in integrated light studies is, and will be, the development of new capabilities to spatially map stellar populations outside of the highest surface brightness galaxy centers. The advent of IFU's has already led to major advances in mapping the inner regions of early-type galaxies (e.g., Kuntschner et al. 2006). To push beyond the effective radius in early-type galaxies, large apertures and superb sky subtraction will allow us to reach substantially lower surface brightnesses than before. In this regard, the nodand-shuffle sky subtraction method (Glazebrook \& Bland-Hawthorn 2001) represents a breakthrugh in sky subtraction accuracy. It is also essential to carefully control scattered light in spectrographs, especially in those situations where a steep light gradient is present (e.g., Rose et al. 2005). 


\subsection{Synthetic Spectral Libraries}

Finally, if multi-population analyses are to be successful, it is essential to reliably model NSAR. To do so will almost certainly require a shift in emphasis from empirically based spectral libraries to those based on model atmospheres and spectrum synthesis. The problem is that Solar neighborhood stars have only a very narrow dispersion in $[\alpha / \mathrm{Fe}]$ at a given $[\mathrm{Fe} / \mathrm{H}]$ (e.g., Reddy, Lambert \& Prieto 2006). This (unfortunate) homogeneity in Galactic abundances makes it impossible to utilize the minimal dispersion in NSAR in the Solar Neighborhood to test for NSAR effects on stellar spectra. Thus while empirical libraries will still play a vital role as benchmarks for the synthetic spectra, the development of such synthetic spectral libraries, which are just coming into the public domain, will provide a major new resource to modellers.

\section{Acknowledgements}

I wish to acknowledge the many stimulating talks and discussions at the symposium, which led to new perspectives in this review. Note that only part of this talk was actually given, due to time limitation!

\section{References}

Burstein, D., Faber, S. M., Gaskell, C. M., \& Krumm, N. 1984, ApJ 287, 586

Caldwell, N., Rose, J. A. \& Cancannon, K. D. 2003, AJ 125, 2891

Coelho, P., Barbuy, B., Melendez, J., Schiavon, R. P. \& Castilho, B. 2005, A\&A 443, 735

Edvardsson, B., Andersen, J., Gustafsson, B., Lambert, D. L., Nissen, P. E. \& Tomkin, J. 1993, A\&S 275,101

Faber, S. M. 1973, ApJ 179, 731

Faber, S. M., Friel, E. D., Burstein, D. \& Gaskell, C. M. 1985, ApJS 57, 71

Faber, S. M., 2005, astro-ph/0506044

Ferreras, I., Pasquali, A., de Carvalho, R. R., de la Rosa, I. G. \& Lahav, O. 2006, MNRAS 370, 828

Gibson, B. K., Madgwick, D. S., Jones, L. A., Da Costa, G. S. \& Norris, J. E. 1999, AJ 118, 1268

Glazebrook, K. \& Bland-Hawthorn, J 2001, PASP 113, 197

Gunn, J. E., Stryker, L. L. \& Tinsley, B. M. 1981, ApJ 249, 48

, Korn, A. J., Maraston, C., \& Thomas, D. 2005, A\& $A$ 438, 685

Kuntschner, H. 2000, MNRAS 315, 184

Kuntschner, H., Lucey, J. R., Smith, R. J., Hudson, M. J. \& Davies, R. L. 2001, MNRAS 323, 615

Kuntschner, H., Smith, R. J., Colless, M., Davies, R. L., Kaldare, R. \& Vazdekis, A. 2002, MNRAS 337, 172

Kuntschner, H. et al. 2006, MNRAS 369, 497

Lee, H.-C., Yoon, S.-J. \& Lee, Y.-W. 2000, AJ 120, 998

Lee, H.-C. \& Worthey, G. 2005, ApJS 160, 176

Madgwick, D. S. et al. 2002, MNRAS 333, 133

Maraston, C. 2005, MNRAS 362, 799

Mengel, J. G., Demarque, P., Sweigart, A. V. \& Gross, P. G. 1979, ApJS 40, 733

Mouhcine, M. \& Lancon, A. 2003, A\& A 402, 425

Nelan, J. E., Smith, R. J., Hudson, M. J., Wegner, G. A., Lucey, J. R., Moore, S. A. W., Quinney, S. J. \& Suntzeff, N. B. 2005, ApJ 632, 137

Nolan, L. A., Dunlop, J. S., Panter, B., Jimenez, R., Heavens, A. F. \& Smith, G. 2006, astro$\mathrm{ph} / 0605417$

O'Connell, R. W. 1976, ApJ 206, 370

O'Connell, R. W. 1980, ApJ 236, 430 
O'Connell, R. W. 1986, in "Stellar Populations", edited by C. A. Norman, A. Renzini, \& M. Tosi, Cambridge Univ. Press, p. 167

Ocvirk, P., Pichon, C., Lancon, A. \& Thiebaut, E. 2006, MNRAS 365, 46

Oke, J. B. 1969, PASP 81, 110

Panter, B., Heavens, A. F. \& Jimenez, R. 2003, MNRAS 343, 1145

Peletier, R. F. 1989, PhD Thesis, University of Groningen

Peterson, R. C. 1976, ApJ 210, L123

Prochaska, L. C., Rose, J. A. \& Schiavon, R. P. 2005, AJ 130, 2666

Prochaska, L. C., Rose, J. A, Caldwell, N., Castilho, B. V., Concannon, K., Harding, P., Morrison, H. \& Schiavon, R. P. 2006, $A J$ (submitted)

Proctor, R. N. \& Sansom, A. E. 2002, MNRAS 333, 517

Prugniel, P. \& Soubiran, C. 2001, A\&SA 369, 1048

Renzini, A. 1986, in "Stellar Populations", edited by C. A. Norman, A. Renzini, \& M. Tosi, Cambridge Univ. Press, p. 213

Reddy, B. E., Lambert, D. L. \& Prieto, C. A. 2006, MNRAS 367, 1329

Robinson, L. B. \& Wampler, E. J. 1972, PASP 84, 161

Rose, J. A. 1985, AJ 90, 1927

Rose, J. A. 1994, AJ 107, 206

Rose, J. A., Arimoto, N., Caldwell, N., Schiavon, R. P., Vazdekis, A. \& Yamada, Y. 2005, AJ 129,712

Sanchez-Blazquez, P., Peletier, R. F., Jimenez-Vicente, J., Cardiel, N., Cenarro, A. J., Falcon-Barroso, J., Gorgas, J., Selam, S. \& Vazdekis, A. 2006a, MNRAS 371, 703

Sanchez-Blazquez, P., Gorgas, J., Cardiel, N. \& Gonzalez, J. J. 2006b, MNRAS 457, 809

Schiavon, R. P., Faber, S. M., Castilho, B. V. \& Rose, J. A. 2002a, ApJ 580, 850

Schiavon, R. P., Faber, S. M., Rose, J. A. \& Castilho, B. V. 2002b, ApJ 580, 873

Schiavon, R. P., Rose, J. A., Courteau, S. \& MacArthur, L. A. 2004, ApJ 608, L33

Schiavon, R. P., Rose, J. A., Courteau, S. \& MacArthur, L. A. 2005, ApJS 160, 163

Singh, H. P., Yuasa, M., Yamamoto, N. \& Gupta, R. 2006, PASJ 58, 177

Spinrad, H. \& Taylor, B. J. 1971, ApJS 22, 445

Spinrad, H., Gunn, J. E., Taylor, B. J., McClure, R. D. \& Young, J. W. 1971, ApJ 164, 11

Tinsley, B. M. 1972, ApJ 178, 319

Thomas, D., Maraston, C. \& Bender, R. 2003, MNRAS 339, 897

Thomas, D., Maraston, C., Bender, R. \& Mendes de Oliveira, C. 2005, ApJ 621, 673

Trager, S. C., Faber, S. M., Worthey, G. \& Gonzalez, J. J. 2000 AJ 120, 165

Trager, S. C., Worthey, G., Faber, S. M. \& Dressler, A. 2005, MNRAS 362, 2

Tripicco, M. J. \& Bell, R. A. 1995, AJ 110, 3035

Valdes, F., Gupta, R., Rose, J. A., Singh, H. P. \& Bell, D. J. 2004, ApJS 152, 251

Vazdekis, A., Peletier, R., Beckman, J. E. \& Casuso, E. 1997, ApJS 111, 203

Vazdekis, A. \& Arimoto, N. 1999, ApJ 525, 144

Vazdekis, A., Salaris, M., Arimoto, N. \& Rose, J. A. 2001, ApJ 549, 274

Wampler, E. J. 1966, ApJ 144, 921

Worthey, G., Faber, S. M. \& Gonzalez, J. J. 1992 ApJ 398, 69 


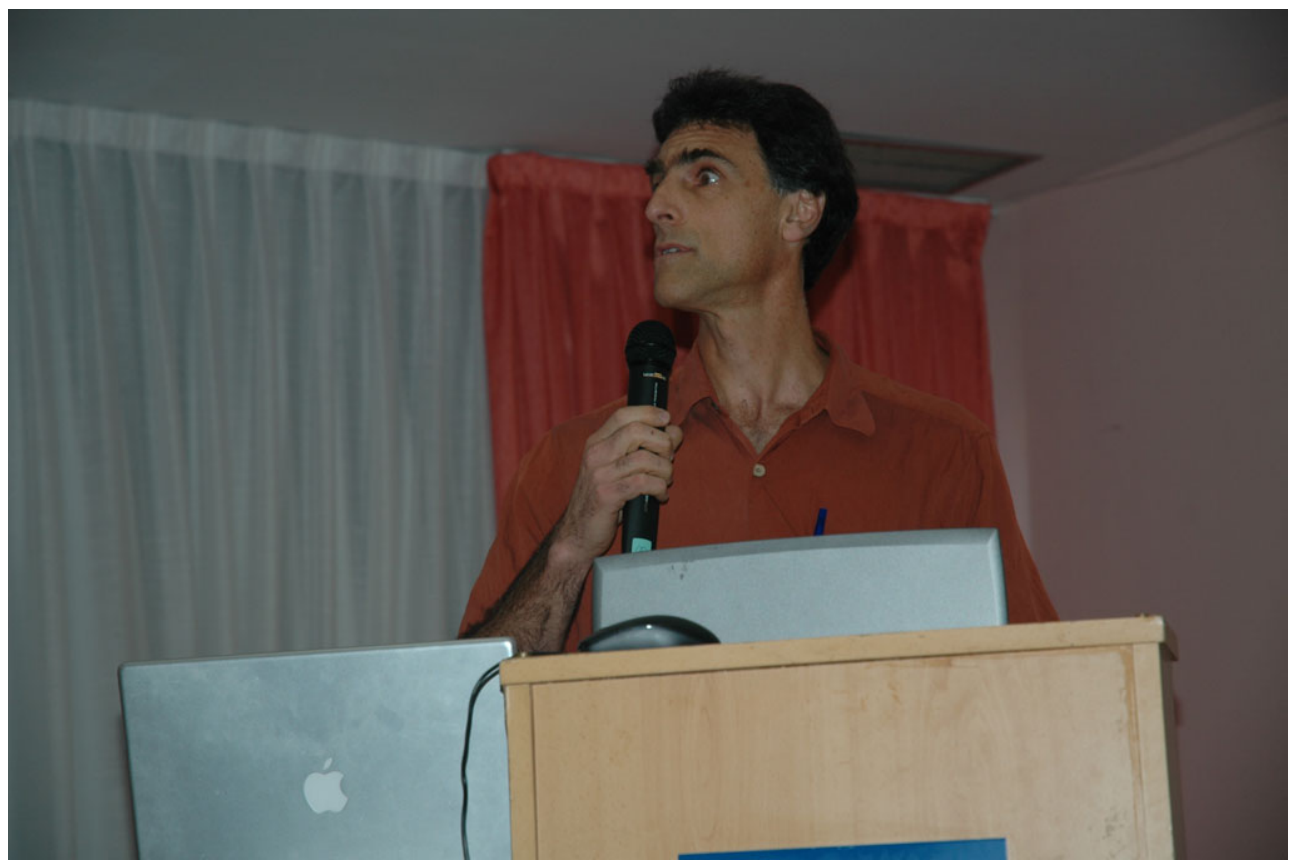

The speaker. 